\section{A device to record gross motor movements in human subjects*}

\author{
DAVID L. ELWOOD $\dagger$ \\ Quinco Consulting Center, Columbus, Indiana 47201
}

A P. R. Mallory Crime Alert CA-2 was used to detect gross motor movements in human Ss. The device was sensitive enough to detect hard eye blinking, rapid movements of a single finger, or more general movements of arms and legs while the $S$ rested in a recliner chair. Noxious audio feedback was effective in inhibiting gross motor movements.

Clinical psychologists often take note of excessive-movement types of behaviors in patients. The patient who drums his fingers on the chair arm, makes his feet dance on the floor, gestures with each word, etc., is often diagnosed as being anxious. Un rortunately, statements concerning the correlation between amount of motor movement and anxiety level have usually been based on impressionistic observations rather than on any kind of quantitative data. The purpose of this article is to describe an instrumentation system developed to quantify the amount of time during which gross motor movements occur in patients.

\section{APPARATUS}

The system was used in a small room measuring $6 \mathrm{ft}$ wide, $7 \mathrm{ft}$ deep, and $7 \mathrm{ft}$ high in which automated psychological testing and treatment procedures were administered. A P.R. Mallory CA-2 Crime Alert device was modified so that the high-pitched sound associated with triggering was omitted; the device was made more sensitive to movement detection, an "on" indicator lamp was disconnected, and the internal relay that closed with triggering was removed and mounted separately in another room (the relay clicked each time the patient moved). The CA-2 was mounted on an adjustable bracket near the ceiling of the treatment-testing room and was aimed toward the patient at about a 45-deg angle. When the CA-2 was turned on, there were no audible sounds or visual cues that indicated to the patient that the device was operating. The relay removed from the CA-2 was interfaced with a general-purpose automated system

*This research was supported by NIMH Research Grant MH 14864.

fSend reprint requests to David $L$. Elwood, Automated Psychology Laboratory, Quinco Consulting Center, 2075 Lincoln Park Drive, Columbus, Indiana 47201. The author thanks Carolyn L. Clark for her assistance in analyzing the data of this report. Also, thanks are due to Chuck Klasing for making internal changes in the CA-2. located in a nearby shop (Elwood \& Griffin, 1972; Elwood, 1972). A general schematic diagram of the circuitry is shown in Fig. 1 . A $-12-\mathrm{V}$ lead was brought into the normally open terminal of the relay and another lead was taken from the common terminal to a Behavior Research Systems CX-205. The normal output from the CX-205 was run into an AND gate (AG-207) along with 10/sec pulses from a precision clock $(\mathrm{MV}-4 \mathrm{~S})$. The output of the AG-207 was then taken into cascaded decade counters (DC-201s) that stored the amount of time during which the patient made gross motor movements.

A second output from the CX-205 was run through a toggle switch and into a click generator (CL-201); from there it went through an audio amplifier (AA-202) and back to a speaker mounted in the treatment-testing room where the movement. patient was being monitored. Thus, it was possible not only to store the times (10ths of seconds) during which patients moved while in the treatment-testing room, but also to provide noxious audio feedback each time the patient executed a detectable movement. The time-movement data stored in the decade counters were printed out on a Teletype each time a new test item was presented or a new step was taken in a treatment program.

The movement measurement system described here was quite sensitive. For example, during psychological treatment applications, patients usually rested in a recliner chair with the CA-2 aimed toward their torsos. With this technique, the CA-2 usually detected hard eye blinking, rapid facial contortions, rapid movements of a single finger as the patient rested his arms on the chair arms, and virtually all gross motor movements involving the torso, arms, legs, and head. The device was especially sensitive to movements in which the patient raised his body or head nearer to the CA-2. On the other hand, the monitor failed to detect foot movements occurring outside of the focus of the device, as well as certain specific motions, e.g., slowly executed head turnings.

\section{SUPPORTING DATA}

Data were collected on 14 patients who participated in automated psychological treatment sessions both with and without noxious audio feedback. It was felt desirable to try to

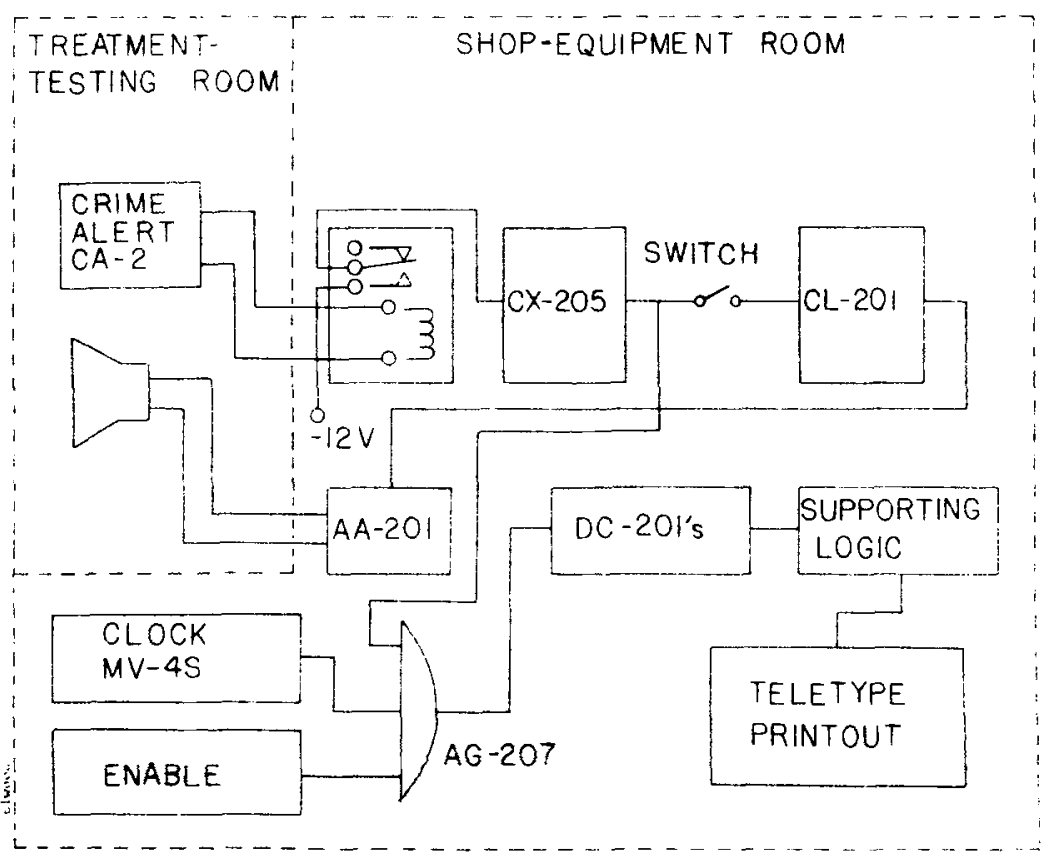

Fig. 1. General schematic diagram of circuitry required for detecting and recording gross motor movements and providing noxious audio feedback for 
inhibit excessive motor movements since these often function as distractors that impair a patient's ability to concentrate during a treatment session. Furthermore, the successful inhibition of movement could be a step in the direction of helping a patient learn to control one aspect of anxious behavior. Correlated means $t$ tests indicated that noxious audio feedback was greatly effective in inhibiting gross motor movements $(t=$ $5.22, \mathrm{df}=13, \mathrm{p}<.001)$. Without feedback, patients moved approximately $13.5 \%$ of time in the treatment-testing room; with feedback, Ss moved only $2.5 \%$.

\section{CONCLUSIONS}

The CA-2 movement measurement device helps to open up for scientific investigation an aspect of clinical behavior often assumed to have importance, but one that is seldom approached quantitatively. The device may be used diagnostically to provide an objective measure of gross motor movement associated with anxiety; it may be used also to measure hyperactivity in children, to assess drug effects, etc. The CA-2 may have treatment applications as well. It is clear from the work in our laboratory that noxious audio feedback can inhibit motor movements. Of course, it remains to be demonstrated that inhibiting motor movement can benefit the patient by increasing concentration or lowering anxiety level.

Finally, the CA-2 can be used as an accurate movement measurement and feedback device without the many interfacing complications described here. The instrument can be modified to record accumulated movement time on an ordinary electric clock and to present auditory feedback (e.g., a buzzer) that operates directly off the $110-\mathrm{V}$ line that powers the clock. The $\mathrm{CA}-2$ inciudes a $110-\mathrm{V}$ ac outlet that is enabled whenever the device is triggered. The Crime Alert (currently available model, CA-1A) sells for $\$ 99.95$.

\section{REFERENCES}

ELWOOD, D. L. Test retest reliability and cost analyses of automated and face to face intelligence testing. International Journal of Man-Machine Studies, 1972, 4 , 1-22.

ELWOOD, D. L., \& GRIFFIN, H. R. Individual intelligence testing without the examiner: Reliability of an automated method. Journal of Consulting \& Clinical Psychology, 1972, 38, 9-14. 